\title{
Traumatic Posterior Atlantoaxial Dislocation without Related Fractures of C1 - C2 in a Patient with Traumatic Brain Injury: Case Report and Literature Review
}

\author{
Lenin Moyo*, Aaron Musara, Kazadi Kalangu \\ Department of Surgery, Neurosurgery Unit, University of Zimbabwe, Harare, Zimbabwe \\ Email address: \\ moyolenin@gmail.com (L. Moyo),musaraaaron@zol.co.zw (A. Musara),kazkal2003@hotmail.com(K. Kalangu) \\ ${ }^{*}$ Corresponding author
}

\section{To cite this article:}

Lenin Moyo, Aaron Musara, Kazadi Kalangu. Traumatic Posterior Atlantoaxial Dislocation without Related Fractures of C1 - C2 in a Patient with Traumatic Brain Injury: Case Report and Literature Review. International Journal of Neurosurgery. Vol. 3, No. 1, 2019 , pp. 5-8. doi: $10.11648 /$ j.ijn.20190301.12

Received: February 6, 2019; Accepted: June 25, 2019; Published: July 9, 2019

\begin{abstract}
It is exceptionally rare to have posterior atlanto axial dislocation without an associated atlas or odontoid fracture and very few cases have been reported in literature. We present a case of a 50 year old female pedestrian who was hit by car while crossing a road. She presented with a depressed level of consciousness and the Glasgow coma score of 12/15, pupils were equal and reactive to light and all limbs were moving equally. She had an extensive abrasion on the forehead which extended into scalp. CT scan showed a posterior dislocation of the atlas with respect of axis with no associated fractures of C 1 and $\mathrm{C} 2$. She had traumatic subarachnoid hemorrhage in the basal cisterns and multiple brainstem contusions. Closed reduction of the dislocation was done successfully under fluoroscopy guidance. MRI was done post reduction because funds were not available initially and it showed increased signal in the tectorial membrane and transverse and alar ligaments with mild cord oedema at the cervicomedullary junction. However, the patient post reduction began to deteriorate and eventually passed away before definitive treatment of her cervical-spine injury. The case is reported in view of its rarity and to review literature on this uncommon condition.
\end{abstract}

Keywords: Posterior, Atlantoaxial, Dislocation

\section{Introduction}

Traumatic upper cervical injuries constitute $20 \%$ of acute cervical spine injuries. However, traumatic atlantoaxial dislocation is infrequent and commonly presents as anterior translational dislocation. Posterior atlantoaxial dislocation without odontoid fracture is extremely rare and often results in fatal spinal cord injury after high-velocity trauma. It is commonly detected at post mortem examination [1]. Very few cases have been reported in literature. We here present a rare case of posterior atlantoaxial dislocation without associated fractures in a head injured patient.

\section{Case Report}

We present a 50 year old female pedestrian who was hit by an automobile while crossing a road and immediately lost consciousness. She presented with depressed level of conscious and was managed as per the Advanced Trauma Life Support protocol. She was hemodynamically stable with mild respiratory distress. She had a Glasgow coma score of 12/15, pupils were equal and reactive to light and had reduced but equal movement in all limbs. Signs of basal skull fracture were present and had forehead abrasion associated with a deep scalp laceration. The rest of the examination was normal.

CT scan showed a posterior dislocation of the atlas with respect to the axis with no associated fractures of $\mathrm{C} 1$ and $\mathrm{C} 2$ (Figures 1 \& 2). 


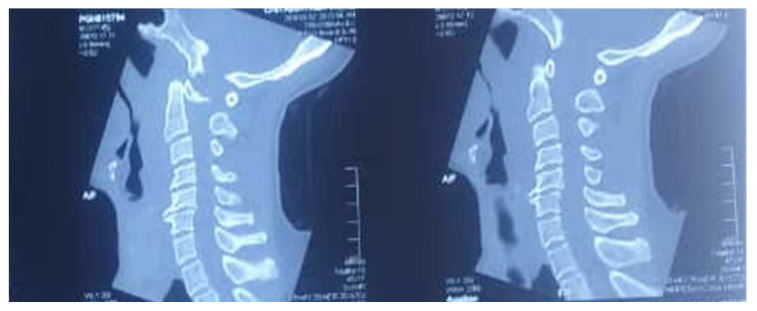

Figure 1. Shows odontoid peg anterior to anterior C1 arch.
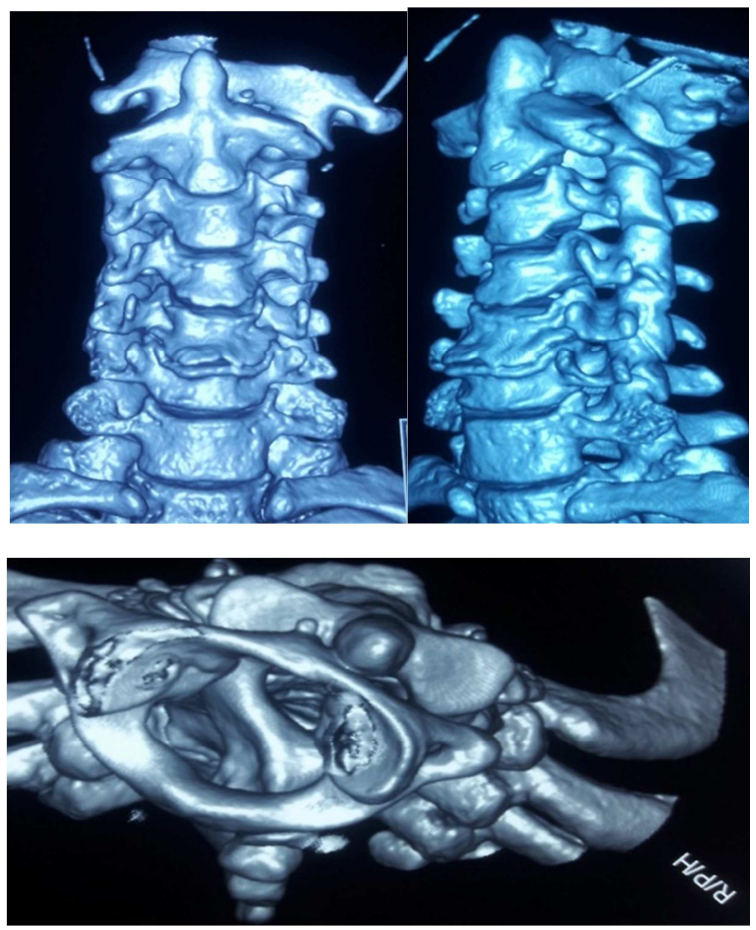

Figure 2. Top left image - AP 3D CT reconstruction showing intact odontoid process anterior to anterior arch of C1, Top right image-lateral $3 D C T$ reconstruction showing dislocation of the atlanto-axial joint. Bottom imageaxial $3 D$ reconstruction showing posterior atlantoaxial dislocation with intact $C 1$ ring.

She had traumatic subarachnoid hemorrhage in the basal cisterns and multiple contusions in the cerebrum, cerebellum and brain stem (Figure 3).

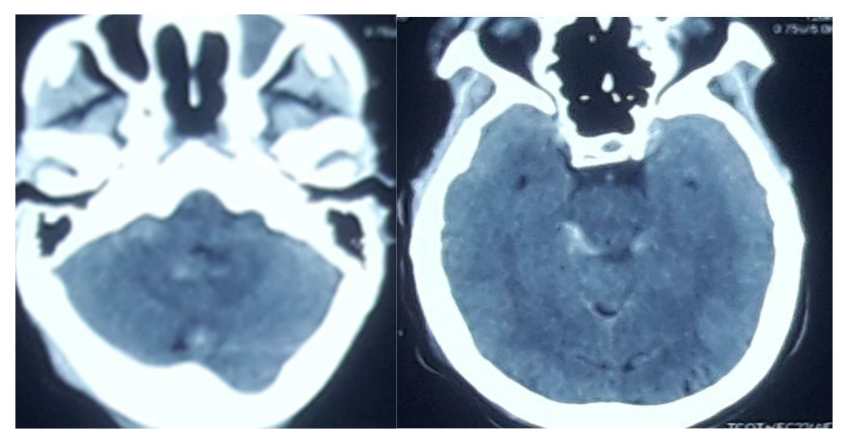

Figure 3. Left image - multiple contusions - cerebellum and brainstem, right image -subarachnoid haemorrhage in the quadrigerminal cisterns.

Closed reduction was done successfully under fluoroscopy guidance (Figure 4).

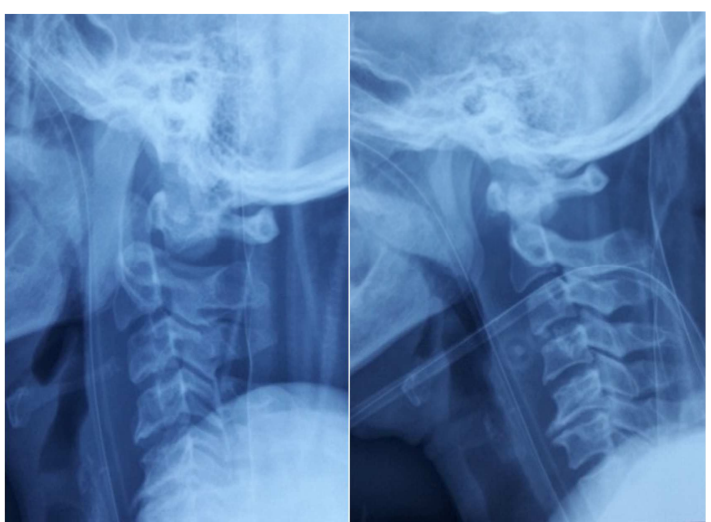

Figure 4. Left - pre reduction with posterior atlantoaxial dislocation, right post reduction.

MRI was done post reduction and showed increased signal in the tectorial membrane and transverse and alar ligaments with mild cord oedema at the cervicomedullary junction (Figure 5).

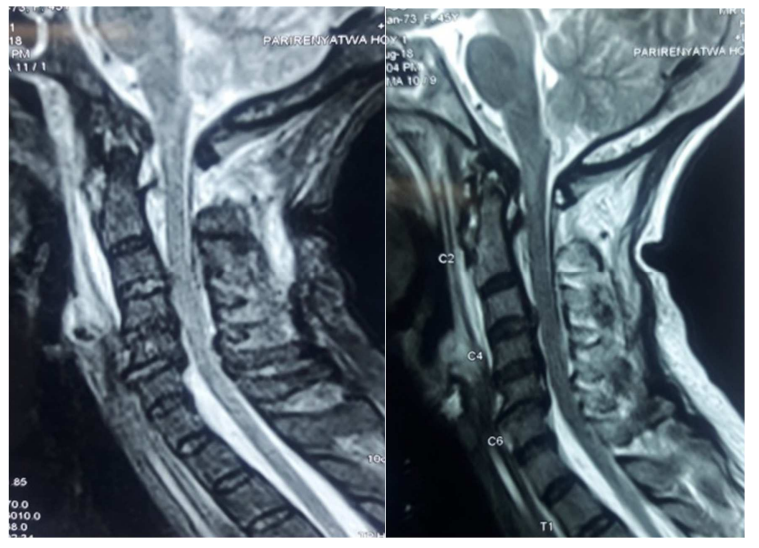

Figure 5. There was ligamentous disruption with oedema at the tectorial, transverse and apical ligaments at the peg and rupture of the posterior longitudinal ligament. Mild oedema at the cervicomedullary junction. Preexisting disc degeneration with possibly disc herniation at C3/C4, C4/C5 and C6/C7 levels.

Unfortunately post reduction patient took a downturn and deteriorated until she died.

\section{Discussion}

\subsection{Anatomy and Biomechanics of the Craniocervical Junction}

The craniocervical junction is the most mobile segment of the spine and contributes to about $40^{\circ}-47^{\circ}$ of motion in the cervical spine. The $\mathrm{C} 0-\mathrm{C} 1$ (occipitoatalantal) articulation is a ball and socket joint and the $\mathrm{C} 1-\mathrm{C} 2$ is a biconvex joint. The atlantoaxial joint accounts for more than $50 \%$ of neck rotation. It also contributes to lateral translation of $3 \mathrm{~mm}$, lateral tilt of about $5^{\circ}$, anteroposterior translation of $3 \mathrm{~mm}$ and flexion-extension of $5^{\circ}-10^{\circ}$. The osseo-ligamentous complex comprising of the atlas, odontoid peg and transverse atlantal ligament is important for stability. The weakest part being the transverse atlantal ligament, so when it ruptures 
dislocation is usual anterior. Posterior dislocation without associated fracture is extremely rare and when it occurs it is associated with an odontoid peg and/or anterior arch of atlas fracture [2, 3].

In a 2015 review paper on post traumatic posterior atlantoaxial dislocation without associated fracture of $\mathrm{C} 1$ or C2 , investigators found 19 cases in both English and none English literature [4].

\subsection{Classification of Atlantoaxial Dislocation}

There are four different classification systems for atlantoaxial dislocation. The parameters considered are reducibility, cause, time to diagnosis and direction of dislocation.

According to reducibility $\mathrm{AAD}$ can be described as reducible, irreducible on non-reducible. Reducible type can be easily reduced by closed dual direction traction. The irreducible type cannot be reduced by traction and requires surgical release of soft tissue which would be preventing reduction. A non-reducible type is due to bony fusion between $\mathrm{C} 1$ \& $\mathrm{C} 2$ and decompression by odontoid peg resection is necessary [5].

Depending on time from dislocation to definite diagnosis it can be classified as fresh type if diagnosis is made within 3 weeks and old type if it's more 3 weeks old. Time to diagnosis can be prognostic on the success of reduction with the fresh type more likely to be reducible.

AAD can also be classified according to the causes which can be congenital, traumatic or pathologic. Trauma is the most common cause of dislocation, it is usually associated with disruption of the atlantoaxial osseoligamentous complex [6].

Classification according to direction of dislocation describe $\mathrm{AAD}$ as anterior, posterior or rotational. The most common being anterior dislocation which is often associated with rupture of the transverse atlantal ligament or malformation of the dens. Relatively uncommon is posterior AAD which is often associated fracture of the dens [7].

The case presented here is a fresh traumatic reducible posterior AAD. It uniquely does not have any associated fractures or congenital abnormalities of the odontoid or atlas. It's possible though that the patient might have had preexisting pathology like connective tissue or an inflammatory disease predisposing her to dislocation.

\subsection{Mechanism of Injury of Posterior Atlantoaxial Dislocation}

Haralson and Boyd were the first ones to report a case of posterior atlantoaxial dislocation without associated odontoid peg fracture. Their hypothesis for the mechanism of injury was a combination of severe hyperextension and distraction. It has not been shown clinically or experimentally but the fact that $68 \%$ of patients have some facial injuries has made many authors to agree with proposed mechanism of injury [8]. Our patient had associated facial injuries which suggest that she might have had some hyper extension during the trauma.

\subsection{Clinical Symptoms and Signs}

Patients may present with neck pain or stiffness, dysphagia or dyspnoea due to compression oesophagus and trachea respectively. About $53 \%$ of patients present with no neurologic deficit and those who have deficits have mild or transient motor weakness. Patients with deficits virtually recover without any residual long term neurologic deficits. The spinal canal sagittal diameter is greatest at the craniocervical junction. According to Steel's rule of thirds at the occipitocervical junction one third of the spinal canal is occupied by the odontoid process, one third by spinal cord and the final third by cerebrospinal fluid. With AAD the spinal cord can displace the surrounding cerebrospinal fluid before it is compressed which explains the large proportion of patients without neurologic deficits $[4,9]$.

\subsection{Diagnosis}

Radiographs have a poor sensitivity of detecting craniocervical junction pathology due to overlap of osseous structures. CT scan with multiplanar reconstruction and 3dimensional reconstruction is always the gold standard in identifying the osseous anatomy and provides excellent visualization of the rare dislocation. MRI is sensitive in detecting the integrity of the critical stabilizing ligaments and the status of the cervical spinal cord [10].

\subsection{Treatment}

Closed reduction is achieved by using dual direction traction. A horizontal axial force is used to apply traction while a perpendicular force is applied to the neck [6]. We successfully reduced the posterior AAD using dual direction traction. We had no access to neuromonitoring which could make procedure safer given that our patient had a depressed level of consciousness.

For reducible AAD the surgical approach of choice is posterior atlantoaxial fixation. Patients with irreducible or non-reducible $\mathrm{AAD}$ will require anterior transoral release or anterior decompression prior to posterior fixation.

\subsection{Posterior Approaches}

Posterior wiring and clamping techniques can only be used in patients with intact posterior structures. Biomechanical wiring techniques have insufficient anti rotation strength hence need a rigid cervical brace until bony fusion is achieved.

Posterior trans-articular screw fixation, the Magerl technique is used in patients with or without intact posterior elements of $\mathrm{C} 1$ and $\mathrm{C} 2$. Biomechanically compared with the wiring techniques, it has a more rigid fixation strength and better anti-rotational ability.

Posterior pedicle screw-rod fixation, commonly known as the Goel - Harms technique, provides much greater biomechanical rigidity strength when compared to wiring or trans-articular screw fixation [11]. 
Posterior Occipital Cervical Fixation has the disadvantage of sacrificing motion at the atlanto occipital joint which may impact negatively on the patient's quality of life. It is however indicated, when there is associated congenital atlanto-occipital fusion, incomplete posterior atlas or atlantooccipital instability [12].

\subsection{Anterior Approaches}

Transoral release is an important technique for dealing with irreducible AAD. Soft tissue scarring behind the anterior arch of the atlas may restrict reduction in these cases. Debriding the soft tissue is important to achieve reduction.

Transoral decompression may be necessary if transoral release fails, this may include odontoid or extensive axis resection. Some cases of non-reducible AAD are due to bone fusion of the lateral masses.

After transoral release and reduction, fixation can be done using screw and plate system. The screws are placed in lateral mass of the atlas lateral mass and in axis vertebra along the pedicles. Anterior cervical trans-articular screw is another alternative but it is usually difficult to put a graft into the facet joint $[6,13-15]$.

\section{Conclusion}

Managing patients with posterior atlantoaxial dislocation without odontoid fracture who also have concomitant traumatic brain injury presents a unique challenge in low resource settings. Having an MRI done before reduction would provide information on the relationship of osseous and neuro-vascular structures and help guide reduction. Considering that our patient's condition worsened after reduction we strongly recommend the use of neuromonitoring during reduction and operation of patients with craniocervical injury associated traumatic brain injury.

\section{References}

[1] Xu Y, Li F, Guan H, Xiong W. Traumatic Posterior Atlantoaxial Dislocation Without Associated Fracture but With Neurological Deficit. Medicine (Baltimore) [Internet]. 2015 Oct 30 [cited 2019 Jun 18]; 94 (43). Available from: https://www.ncbi.nlm.nih.gov/pmc/articles/PMC4985386/

[2] Lopez AJ, Scheer JK, Leibl KE, Smith ZA, Dlouhy BJ, Dahdaleh NS. Anatomy and biomechanics of the craniovertebral junction. Neurosurg Focus. 2015 Apr; 38 (4): E2.
[3] Salunke P. Congenital Atlantoaxial Dislocation: Nature's Engineering Gone Wrong and Surgeon's Attempt to Rectify It. J Pediatr Neurosci. 2018; 13 (1): 1-7.

[4] Hu D, Yang X, Wang J. Traumatic Posterior Atlantoaxial Dislocation Without Fracture of Odontoid Process: A Case Report and Systematic Analysis of 19 Cases. J Orthop Trauma. 2015 Sep; 29 (9): e342-345.

[5] Deepak AN, Salunke P, Sahoo SK, Prasad PK, Khandelwal NK. Revisiting the differences between irreducible and reducible atlantoaxial dislocation in the era of direct posterior approach and C1-2 joint manipulation. J Neurosurg Spine. 2017 Mar; 26 (3): 331-40.

[6] Yin Q, Wang J. Current Trends in Management of Atlantoaxial Dislocation. Orthop Surg. 2015 Aug; 7 (3): 189-99.

[7] Meyer C, Eysel P, Stein G. Traumatic Atlantoaxial and Fracture-Related Dislocation [Internet]. BioMed Research International. 2019 [cited 2019 Jun 18]. Available from: https://www.hindawi.com/journals/bmri/2019/5297950/

[8] Yu HM, Malhotra K, Butler JS, Wu SQ. Anterior and posterior fixation for delayed treatment of posterior atlantoaxial dislocation without fracture. BMJ Case Rep. 2015 Oct 29; 2015.

[9] Lacy J, Gillis CC. Atlantoaxial Instability. In: StatPearls [Internet]. Treasure Island (FL): StatPearls Publishing; 2019 [cited 2019 Jun 18]. Available from: http://www.ncbi.nlm.nih.gov/books/NBK519563/

[10] Offiah CE, Day E. The craniocervical junction: embryology, anatomy, biomechanics and imaging in blunt trauma. Insights Imaging. 2017 Feb; 8 (1): 29-47.

[11] Huang D-G, Hao D-J, He B-R, Wu Q-N, Liu T-J, Wang X-D, et al. Posterior atlantoaxial fixation: a review of all techniques. Spine J Off J North Am Spine Soc. 2015 Oct 1; 15 (10): 2271-81.

[12] Kukreja S, Ambekar S, Sin AH, Nanda A. Occipitocervical Fusion Surgery: Review of Operative Techniques and Results. J Neurol Surg Part B Skull Base. 2015 Sep; 76 (5): 331-9.

[13] Polli FM, Miscusi M, Forcato S, Raco A. Atlantoaxial anterior transarticular screw fixation: a case series and reappraisal of the technique. Spine J Off J North Am Spine Soc. 2015 Jan 1; 15 (1): 185-93.

[14] Du JY, Aichmair A, Kueper J, Wright T, Lebl DR. Biomechanical analysis of screw constructs for atlantoaxial fixation in cadavers: a systematic review and meta-analysis. J Neurosurg Spine. 2015 Feb; 22 (2): 151-61.

[15] Chun DH, Yoon DH, Kim KN, Yi S, Shin DA, Ha Y. Biomechanical Comparison of Four Different Atlantoaxial Posterior Fixation Constructs in Adults: A Finite Element Study. Spine. 2018 01; 43 (15): E891-7. 